\title{
PHARMACOGENOMICS: THE PROMISE OF CUSTOM-MADE PSYCHIATRY?
}

\author{
R. Correia ${ }^{1}$ \\ 1- Centro Hospitalar Universitário do Algarve, Departamento de Psiquiatria e Saúde Mental, Faro, Portugal. \\ saraivacorreia@gmail.com
}

\section{OBJECTIVES:}

Brief introduction to pharmacogenomics analysis in psychiatry.

\section{BACKGROUND:}

Pharmacogenomics analysis is the scientific methodology that allows the psychiatrist the prediction of the pharmacological response of an individual user to a certain drug therapy, depending on the genetic makeup of each individual. The use of pharmacogenomics analyses allows an individualized and custom-made psychiatry that results in a more assertive and safe psychopharmacological management in terms of drug choice, dosage and the reduction of its side effects. Although the use of pharmacogenomics techniques by physicians is still scarce, its role will be vital in a good medical practice, especially in Psychiatry.

\section{VARIABILITY:}

Differences: Age, Race, BMI, Sex, Diseases, Drugs, Social and Genetic factors

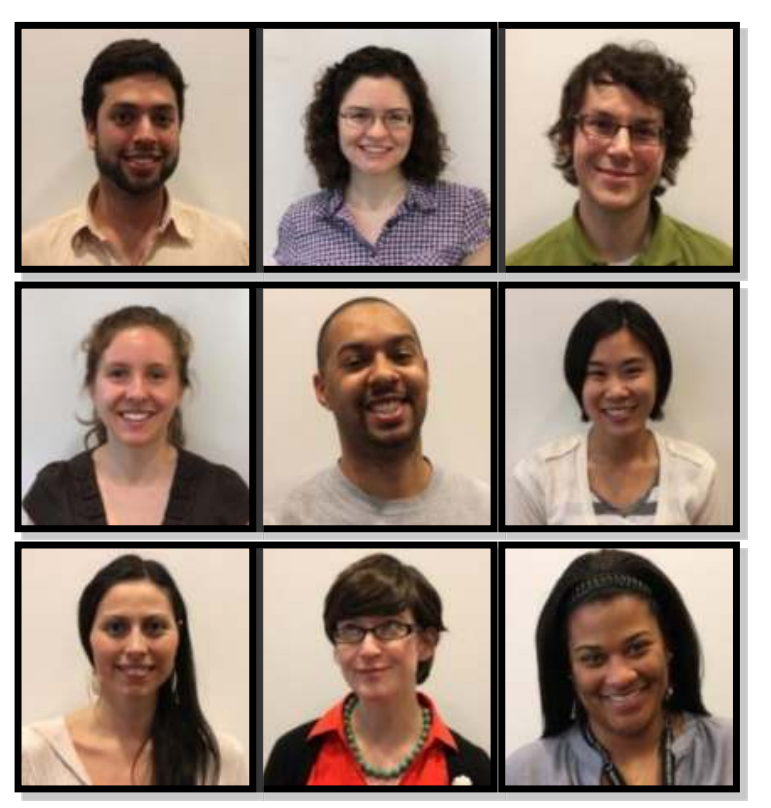

Translate on the different ways we react to drugs: -2x the dosage for the medication to be effective -Some drugs don' $t$ work

-Differences in terms of side effects or toxicity

\section{PERSONALIZED MEDICINE:}

The right drug, in the right dosage, in the right time and in the right patient

\section{APPLICATIONS}

There are some gene variations with known pharmacogenomic effects, like the Cytochrome P450 enzymes (essential for the metabolism of many medications).

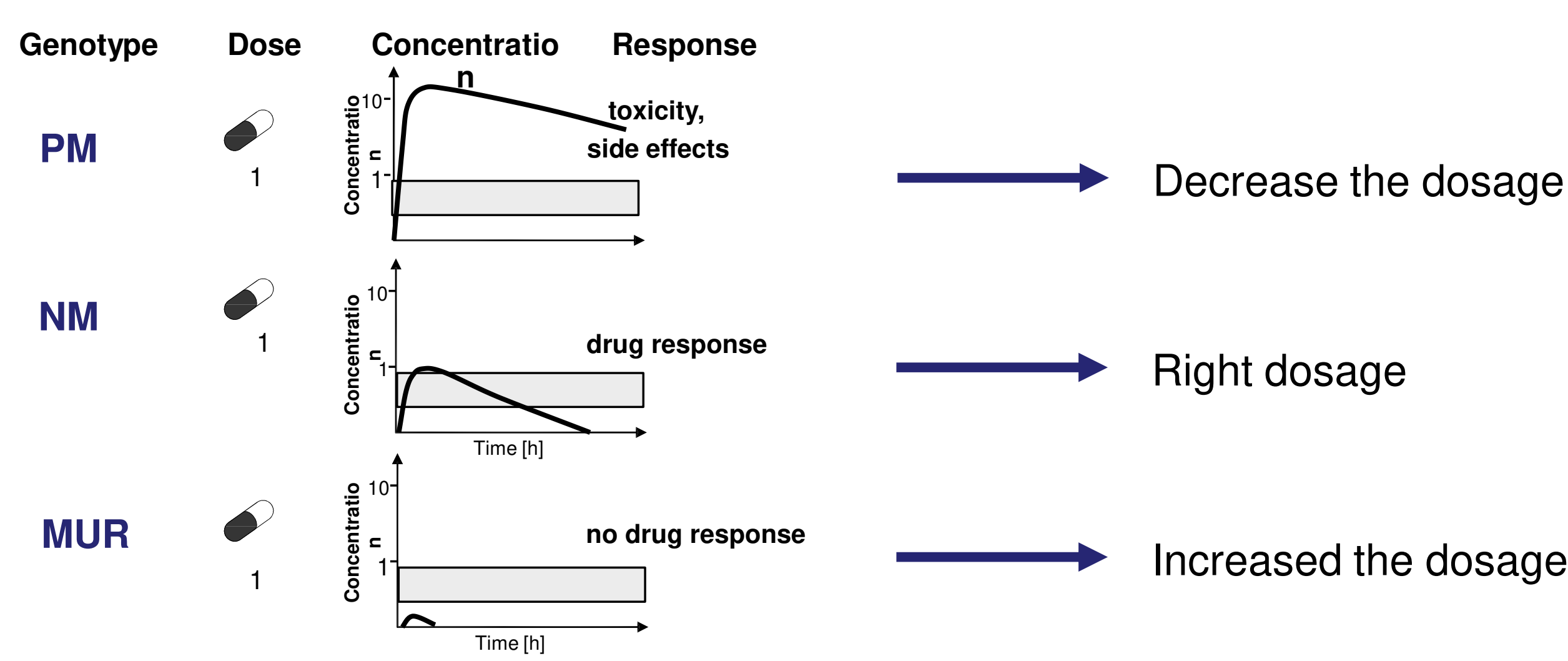

CONCLUSIONS:

The true long-term potential and aim of pharmacogenomics is to design individualized therapy strategies and implement preventive measures based on individual genetic risk factors. At the moment, its use is still emerging due to factors such as its complexity, price and to an wait-and-see attitude by some clinicians. 